\title{
The case for routine screening for SARS-CoV-2 before surgery
}

\author{
Paul Tenenbein, MD, FRCPC $\cdot$ Sheila Riazi, MD, FRCPC $\cdot$ Jennie Johnstone, MD, FRCPC $\cdot$ Shaf Keshavjee, MD, \\ FRCPC $\cdot$ Keyvan Karkouti, MD, FRCPC, MSc $®$
}

Received: 11 May 2020/ Accepted: 24 May 2020/Published online: 3 June 2020

(C) Canadian Anesthesiologists' Society 2020

Severe acute respiratory syndrome coronavirus 2 (SARSCoV-2) has caused more than 5 million cases of coronavirus disease (COVID-19) worldwide and 345,000 deaths as of 25 May 2020. Based on accumulating international experience of the severe impact of the pandemic on susceptible populations and healthcare systems, hospitals in Canada ceased all non-urgent activity to create capacity and to conserve resources for the anticipated surge of patients with COVID-19. On 19 March 2020, the Ontario Chief Medical Officer of Health formally ordered that all non-essential medical services cease. By 28 April 2020, the Financial Accountability Office of Ontario reported that 52,700 hospital procedures had been cancelled. Given the large and growing backlog of patients awaiting medically necessary elective surgery,

P. Tenenbein, MD, FRCPC $\cdot$ S. Riazi, MD, FRCPC Department of Anesthesia and Pain Management, University Health Network, Sinai Health System, Women's College Hospital, University of Toronto, Toronto, ON, Canada

J. Johnstone, MD, FRCPC

Department of Laboratory Medicine and Pathobiology, Sinai Health System, University of Toronto, Toronto, ON, Canada

S. Keshavjee, MD, FRCPC

Department of Surgery, University Health Network, University of Toronto, Toronto, ON, Canada

K. Karkouti, MD, FRCPC, MSc ( $)$

Department of Anesthesia and Pain Management, University

Health Network, Sinai Health System, Women's College

Hospital, University of Toronto, Toronto, ON, Canada

e-mail: Keyvan.karkouti@uhn.ca

Department of Anesthesia and Pain Management, Toronto General Hospital, 200 Elizabeth Street 3EN, Toronto, ON M5G 2C4, Canada there is now an urgent need to ramp up surgical activities across the country. How to safely restart surgical programs in the context of ongoing community spread and localized outbreaks of COVID-19 remains unknown. Herein, we focus on one specific aspect of this question, namely whether all surgical patients should, in addition to detailed clinical screening (i.e., exposure risk and symptoms) for COVID-19, undergo routine preoperative testing for SARS-CoV-2 with nasopharyngeal swabbing and nucleicacid-based testing.

In general, the purpose of routine preoperative testing is to diagnose subclinical or preclinical conditions that could adversely affect patient outcomes unless the test is acted upon to modify the condition, surgery, or both. To be of value, screening tests should be actionable, accurate, and feasible. ${ }^{1}$ When examined according to these criteria, however, most preoperative screening tests perform poorly. ${ }^{1,2}$ Why, then, would preoperative testing for SARS-CoV-2 be any different?

The first and most important consideration is whether patients who undergo surgery during the incubation (or preclinical) period of SARS-CoV-2 infection are at increased risk for adverse postoperative outcomes. Coronavirus disease is a new disease and its impact on postoperative outcomes requires extensive investigation and long-term follow-up; however, early experience suggests that patients who undergo surgery during the incubation period of the disease and then progress to symptomatic infection have a very poor prognosis. In one published case series from China, of the 34 patients who underwent primarily moderate-risk surgery during the incubation period of COVID-19, $44 \%$ needed intensive care unit care and $20 \%$ died. $^{3}$ While this is a relatively small retrospective study and its results may not be necessarily generalizable, the findings are quite plausible 
given what is known about the risk that COVID-19 poses to patients with impaired immune response, ${ }^{4}$ and the immunomodulatory effects of major surgery and anesthesia that predispose patient to immune-mediated complications. ${ }^{5}$

The second consideration is whether the test is actionable. In the case of a positive nasopharyngeal swab, the answer is clearly yes. Given the risk that COVID-19 poses to surgical patients, it would be advisable to defer any procedure that could safely be delayed or consider non-surgical treatment options where appropriate in any patient who tests positive for SARS-CoV-2. If surgery cannot be deferred and suitable alternatives to surgery are not available, a positive test result would allow for a fully informed consent process including recognition of the increased risks. If a decision is made to proceed, it would be prudent to conduct the least invasive form of surgery possible to minimize any consequent physiologic insults on the patient. In addition to informing the decision to proceed with surgery, the test results will also influence the delivery of care. Most surgical procedures require intubation/extubation and other aerosol generating medical procedures that increase risk of nosocomial transmission to healthcare workers taking care of the patient during and after surgery. ${ }^{6}$ A positive test would, therefore, allow for appropriate planning and execution of infection control precautions to prevent caregivers and other patients from becoming infected.

A more challenging question for health systems is, "Is the test accurate enough to alter patient management and infection control precautions based on its results?" Nucleic-acid-based assays such as reverse transcriptase polymerase chain reaction have excellent sensitivity and specificity in laboratory validation studies but the sensitivity and specificity in diagnosing clinical infection with SARS-CoV-2 has yet to be determined. The sensitivity of the test is commonly cited to be around 60$70 \%$, meaning that $30-40 \%$ of patients with the disease will be falsely reported as not having the disease. These results, however, were derived from the early experience with SARS-CoV-2 in small groups of patients, ${ }^{7}{ }^{9}$ and testing accuracy varies based on time from exposure and testing methodology. ${ }^{10}$ Nevertheless, while significant gaps exist in our understanding of the role of nucleic-acid-based testing for ruling out COVID-19 in asymptomatic patients, ${ }^{11}$ it is increasingly clear that it can be used to detect the disease in infected asymptomatic patients. ${ }^{12,13}$ Even if the test has only a modest positive predictive value in asymptomatic patients, it would still be of net benefit since any action taken will simply delay surgery until clinical infection has run its course (in true positive cases) or repeat testing is negative (in false positive cases). In line with this view, in a recent joint statement of the American
Society of Anesthesiologists and the Anesthesia Patient Safety Foundation, routine preoperative testing for SARSCoV-2 was recommended in areas with local disease activity. ${ }^{14}$

Another consideration is the feasibility of the test. Nucleic-acid-based testing is a well-established, commonly available test, and nasopharyngeal swabbing is technically easy to perform and is relatively non-invasive. While testing facilities and supplies were scarce in the early stages of the pandemic, the situation continues to improve across Canada. Ontario, which had one of the lowest percapita testing rates amongst the provinces, for example, has rapidly increased its testing capacity and can now conduct more than 16,000 tests daily. ${ }^{15}$ This capacity should be more than adequate for testing at least every patient who is booked for major surgery, particularly now that surgical volumes are substantially reduced. It is our hope that testing capacity will continue to increase in line with increasing surgical volumes over the next few months.

The COVID-19 pandemic is possibly a once in a century event, but when considering the value of screening for SARS-CoV-2 in asymptomatic surgical patients, it is informative to put it in the context of our response to other infectious pathogens, such as methicillin-resistant Staphylococcus aureus (MRSA). Routine preoperative screening for MRSA has been the standard of care in Canada for several years and is considered cost effective. There can be no argument that the societal costs of MRSA pale in comparison with those of SARS-CoV-2. The public health measures needed to "flatten the curve" have had crippling financial consequences. It is estimated that the Canadian gross domestic product has plunged $10 \%$ while unemployment has ballooned to upwards of $15 \%$, yet preoperative MRSA testing continues while we debate SARS-CoV-2 testing. The stakes will likely continue to grow if we allow continued nosocomial spread of SARSCoV-2. There have already been multiple outbreaks in Toronto hospitals with devastating consequences.

At our hospitals (University Health Network, Sinai Health System, and Women's College Hospital, which are academic health science centres in Toronto, ON, Canada), we instituted routine preoperative testing for SARS-CoV-2 on 23 March 2020, soon after community spread of the virus was suspected. This entailed nasopharyngeal swabbing of all patients within 24-72 hr of surgery, followed by self-isolation until their surgery. Our laboratory turnaround time is less than $24 \mathrm{hr}$, which means that the COVID-19 status of all patients is known before surgery. If the test is positive, non-emergent surgery is delayed and consultation with the infectious diseases service is sought to clear the patient for later surgery. If the test is negative, surgery proceeds and infection control precautions are modified depending on the type of surgery. 
For example, for surgeries considered at low risk for aerosolization (e.g., cardiac surgery), droplet precautions are instituted for all aspects of surgery except for intubation and extubation (during which the anesthesiologist and assistant wear N95 masks and all others leave the operating room). To date, we have identified at least eight asymptomatic patients who tested positive and their surgery was delayed. We do not know if all of these tests were true positives, so we cannot know with certainty how many of them would have had poor outcomes had they proceeded to surgery, nor do we know how many other patients and healthcare workers they would have infected. Nevertheless, identification of this many patients in the short time that the program has been operational confirms the underlying assumptions for instituting routine preoperative testing and highlights the value of this practice.

During the early stages of the pandemic, screening surgical patients for contact history and symptoms was deemed to be appropriate. Now that community spread is well-documented and upwards of $45 \%$ of infected patients are asymptomatic (or pre-symptomatic), ${ }^{12,13}$ relying only on contact history and symptoms for screening of asymptomatic surgical patients is no longer sufficient. How many patients harbouring SARS-CoV-2 should be admitted to our hospitals for elective surgery? We propose that this should be a "never event," so even a single such case would be unacceptable. Implementing routine preoperative testing, while not foolproof, will get us closer to that goal. It is our opinion, therefore, that until such time that the community spread of SARS-CoV-2 ceases, all surgical patients should be routinely screened for the virus before their surgery.

\section{Pour un dépistage systématique du SARS-CoV- 2 avant une chirurgie}

Le coronavirus 2 avec syndrome respiratoire aigu sévère (SARS-CoV-2) a provoqué plus de cinq millions de cas d'infections (COVID-19) dans le monde et, au 25 mai 2020, le nombre global de décès s'élevait à 345 000. En se fondant sur les expériences internationales cumulatives de l'impact majeur de la pandémie sur les populations susceptibles et les systèmes de soins de santé, les hôpitaux canadiens ont suspendu toutes les activités non urgentes afin de disposer de la capacité et des ressources nécessaires pour prendre en charge la hausse anticipée du nombre de patients atteints de la COVID-19. Le 19 mars 2020, le médecin hygiéniste en chef de l'Ontario a formellement décrété la suspension de tous les services médicaux non essentiels. Au 28 avril 2020, le Bureau de la responsabilité financière de l'Ontario rapportait l'annulation de 52700 interventions hospitalières. Étant donné la liste toujours croissante de patients en attente d'une chirurgie non urgente mais nécessaire d'un point de vue médical, il est aujourd'hui urgent d'accélérer les activités chirurgicales partout au pays. En revanche, nous ne savons pas encore comment relancer les programmes chirurgicaux de façon sécuritaire dans le contexte d'une transmission communautaire toujours présente et d'éclosions locales de COVID-19. Dans cet éditorial, nous nous intéressons à un aspect en particulier de cette question : faudrait-il faire passer un test préopératoire systématique pour dépister le SARS-CoV-2 à l'aide d'un écouvillon nasopharyngé et d'un test d'amplification des acides nucléiques à tous les patients chirurgicaux, en plus du dépistage clinique détaillé (c.-à-d. risque d'exposition et symptômes)?

En règle générale, l'objectif des tests préopératoires systématiques est de diagnostiquer les conditions infracliniques ou précliniques qui pourraient avoir un impact négatif sur les devenirs des patients, à moins que des mesures ne soient prises en fonction des résultats du test afin de modifier la condition du patient, sa chirurgie, ou les deux. Pour être utiles, les tests de dépistage doivent être à la fois concrets, précis et réalisables. ${ }^{1}$ Lorsqu'examinés selon ces critères toutefois, la plupart des tests de dépistage préopératoires offrent une performance médiocre. ${ }^{1,2}$ Pour quelle raison le test préopératoire du SARS-CoV-2 serait-il donc différent?

La première question - et la plus importante - est de déterminer si les patients subissant une chirurgie pendant la période d'incubation (ou préclinique) d'une infection au SARS-CoV-2 courent un risque plus élevé de complications postopératoires. Le coronavirus est une pathologie nouvelle, et son impact sur les devenirs postopératoires requiert des recherches approfondies et un suivi à long terme; les premières expériences laissent toutefois penser que le pronostic des patients subissant une chirurgie en période d'incubation de la maladie et qui progressent ensuite à la phase d'infection symptomatique est particulièrement mauvais. Dans une série de cas publiée provenant de Chine, parmi les 34 patients ayant subi une chirurgie de risque principalement modéré pendant la période d'incubation de la COVID-19, $44 \%$ ont requis des soins à l'unité de soins intensifs, et $20 \%$ des patients sont décédés. ${ }^{3}$ Certes, il s'agit d'une étude rétrospective de taille relativement petite et ses résultats pourraient ne pas être généralisables; ces conclusions sont néanmoins plutôt plausibles au vu de nos connaissances quant au risque que la COVID-19 pose aux patients ayant une réponse immunitaire altérée, ${ }^{4}$ ainsi qu'en raison des effets 
immunomodulateurs d'une chirurgie majeure et de l'anesthésie prédisposant les patients à des complications à médiation immunitaire. ${ }^{5}$

La deuxième considération est de savoir si le test a des conséquences concrètes. Dans le cas d'un écouvillon nasopharyngé positif, la réponse est clairement oui. Étant donné le risque que la COVID-19 pose aux patients chirurgicaux, il est conseillé de remettre toute intervention qui peut être retardée en toute sécurité ou d'envisager des options thérapeutiques non chirurgicales, le cas échéant, pour tout patient positif au SARS-CoV-2. Si la chirurgie ne peut être retardée et qu'il n'existe pas d'alternatives adaptées à la chirurgie, un résultat de test positif permettrait l'instauration d'un processus de consentement pleinement éclairé comprenant la reconnaissance des risques accrus. Si la décision d'aller de l'avant avec la chirurgie est prise, il serait prudent de retenir la forme de chirurgie la moins invasive possible afin de minimiser toute insulte physiologique subséquente chez le patient. En plus d'informer la décision d'aller de l'avant avec la chirurgie, les résultats d'un tel test influenceront également la fourniture des soins. La plupart des interventions chirurgicales nécessitent une intubation/extubation et d'autres interventions médicales générant des aérosols, ce qui augmente le risque de transmission nosocomiale aux travailleurs de la santé qui prennent soin du patient pendant et après la chirurgie. ${ }^{6}$ Un test positif permettrait donc une planification adaptée et la mise en œuvre de précautions de contrôle des infections afin d'éviter que les fournisseurs de soins et les autres patients ne soient infectés.

Une question plus difficile pour les systèmes de santé est de savoir si, en se fondant sur son résultat, le test est suffisamment précis pour altérer la prise en charge du patient et les précautions de contrôle des infections. Les tests d'amplification des acides nucléiques, tels que l'amplification par la polymérase avec transcription inverse, ont une sensibilité et une spécificité excellentes dans les études de validation en laboratoire, mais leur sensibilité et leur spécificité pour le diagnostic de l'infection clinique au SARS-CoV-2 n'ont pas encore été déterminées. La sensibilité du test est souvent évaluée aux environs de 60-70\%, ce qui signifie que 30-40\% des patients atteints de la maladie auront des résultats négatifs. Ces résultats sont toutefois dérivés des premières expériences avec le SARS-CoV-2 dans de petits groupes de patients, ${ }^{7}-{ }^{9}$ et la précision du test varie en fonction du délai depuis l'exposition et de la méthodologie de test. ${ }^{10}$ Cependant, alors qu'il existe d'importantes lacunes dans notre compréhension du rôle du test d'amplification des acides nucléiques pour exclure la présence de COVID-19 chez les patients asymptomatiques, ${ }^{11}$ il devient de plus en plus clair que ce test peut être utilisé pour dépister la maladie chez les patients asymptomatiques infectés. ${ }^{12,13}$
Même si le test ne dispose que d'une valeur prédictive positive modeste chez les patients asymptomatiques, il demeurerait néanmoins clairement avantageux étant donné que tout geste posé retarderait simplement la chirurgie jusqu'à ce que l'infection clinique ait suivi son cours (dans le cas d'un vrai positif) ou qu'un test subséquent soit négatif (dans le cas d'un faux positif). Conformément à ce point de vue, dans une déclaration conjointe de l'American Society of Anesthesiologists et de l'Anesthesia Patient Safety Foundation, le dépistage préopératoire systématique du SARS-CoV-2 a été recommandé dans les zones où la maladie est active au niveau local. ${ }^{14}$

Une autre considération touche à la faisabilité du test. Le test d'amplification des acides nucléiques est un test bien établi et aisément disponible, et l'écouvillonnage nasopharyngé est facile à réaliser d'un point de vue technique tout en étant peu invasif. Alors que les centres de dépistage et les tests étaient peu disponibles au début de la pandémie, la situation continue de s'améliorer partout au Canada. Par exemple l'Ontario, qui affiche l'un des taux de dépistage les plus bas parmi les provinces, a rapidement augmenté sa capacité de dépistage et peut aujourd'hui réaliser plus de 16000 tests par jour. ${ }^{15}$ Cette capacité devrait être plus que suffisante pour au minimum tester chaque patient devant subir une chirurgie majeure, particulièrement au vu de la réduction considérable des volumes chirurgicaux. Nous espérons que la capacité de dépistage continuera de s'améliorer au fur et à mesure que les volumes chirurgicaux augmenteront au cours des mois à venir.

La pandémie de la COVID-19 est peut-être un événement exceptionnel, mais lorsqu'on considère l'utilité du dépistage du SARS-CoV-2 chez les patients chirurgicaux asymptomatiques, il est instructif de la comparer à notre réponse à d'autres pathogènes infectieux, comme par exemple le staphylocoque doré résistant à la méthicilline (SARM). Au Canada, le dépistage préopératoire systématique du SARM fait partie de la norme de soins depuis des années et est considéré comme un test rentable. Il est indubitable que les coûts sociétaux du SARM font pâle figure à côté de ceux du SARS-CoV-2. Les mesures de santé publique nécessaires à « aplanir la courbe » ont eu des conséquences financières dévastatrices. On estime que le produit intérieur brut canadien a plongé de $10 \%$, alors que le chômage a augmenté au-dessus des $15 \%$, et pourtant le dépistage préopératoire du SARM se poursuit alors qu'on débat encore du dépistage du SARS-CoV-2. Les enjeux continueront de croître si nous permettons à la transmission nosocomiale du SARS-CoV-2 de se poursuivre. Plusieurs éclosions ont déjà eu lieu dans des hôpitaux de Toronto, avec des conséquences dévastatrices. 
Dans nos hôpitaux (c.-à-d. le University Health Network, le Sinai Health System, et le Women's College Hospital, qui sont des centres de sciences de la santé universitaires à Toronto, ON, Canada), nous avons mis en place un dépistage préopératoire systématique du SARSCoV-2 le 23 mars 2020, peu après que les autorités ont émis la suspicion d'une transmission communautaire du virus. Cela a entraîné l'écouvillonnage nasopharyngé de tous les patients dans les 24-72 h précédant leur chirurgie, suivi d'un auto-isolement jusqu'à la chirurgie. Le temps de traitement de notre laboratoire est de moins de $24 \mathrm{~h}$, ce qui signifie que le statut COVID-19 de tous les patients est connu avant la chirurgie. Si le test est positif, les chirurgies non urgentes sont retardées et une consultation avec le service des maladies infectieuses est organisée afin de permettre au patient d'avoir sa chirurgie plus tard. Si le test est négatif, la chirurgie a lieu et les précautions en matière de contrôle des infections sont modifiées en fonction du type de chirurgie. Par exemple, dans le cas de chirurgies dont le risque d'aérosolisation est considéré comme faible (par ex., une chirurgie cardiaque), les précautions contre les gouttelettes sont instituées pour tous les aspects de la chirurgie sauf l'intubation et l'extubation (pendant lesquelles l'anesthésiologiste et son assistant portent des masques N95 et tous les autres intervenants quittent la salle d'opération). À ce jour, nous avons identifié au moins huit patients asymptomatiques dont le test était positif et dont la chirurgie a été subséquemment retardée. Nous ne savons pas si tous ces tests étaient de vrais positifs, c'est pourquoi nous ne pouvons déterminer avec certitude combien d'entre eux auraient subi des complications si la chirurgie avait eu lieu, ni combien d'autres patients et travailleurs de la santé auraient été infectés. Toutefois, l'identification d'autant de patients dans le court intervalle depuis la mise en place de notre programme confirme les hypothèses sousjacentes à l'instauration du dépistage préopératoire systématique et confirme l'utilité de cette pratique.

$\mathrm{Au}$ début de la pandémie, le dépistage des patients chirurgicaux pour connaître leurs antécédents de contact et leurs symptômes était considéré comme une mesure adaptée. Maintenant que la transmission communautaire est bien documentée et que plus de $45 \%$ des patients infectés sont asymptomatiques (ou présymptomatiques), ${ }^{12,13}$ il ne suffit plus de s'appuyer exclusivement sur les antécédents de contact et les symptômes des patients pour le dépistage des patients chirurgicaux asymptomatiques. Combien de patients atteints de SARS-CoV-2 devrions-nous admettre dans nos hôpitaux pour des chirurgies non urgentes? Selon nous, la réponse devrait être «aucun », et un seul cas serait déjà inacceptable. La mise en œuvre d'un dépistage préopératoire systématique, bien que non infaillible, nous rapprochera de cet objectif. Ainsi, nous pensons que jusqu'à ce que la transmission communautaire du SARSCoV-2 cesse, tous les patients chirurgicaux devraient être systématiquement testés pour exclure la maladie avant leur chirurgie.

\section{Disclosures None.}

Funding statement None.

Editorial responsibility This submission was handled by Dr. Gregory L. Bryson, Deputy Editor-in-Chief, Canadian Journal of Anesthesia.

Déclarations Aucune.

Déclaration de financement Aucune.

Responsabilité éditoriale Cet article a été traité par Dr Gregory L. Bryson, rédacteur en chef adjoint, Journal canadien d'anesthésie.

\section{References}

1. Turnbull JM, Buck $C$. The value of preoperative screening investigations in otherwise healthy individuals. Arch Intern Med 1987; 147: 1101-5.

2. O'Neill F, Carter E, Pink N, Smith I. Routine preoperative tests for elective surgery: summary of updated NICE guidance. BMJ 2016; DOI: https://doi.org/10.1136/bmj.i3292.

3. Lei $S$, Jiang $F, S u W$, et al. Clinical characteristics and outcomes of patients undergoing surgeries during the incubation period of COVID-19 infection. EClinicalMedicine 2020; DOI: https://doi. org/10.1016/j.eclinm.2020.100331.

4. Shi $Y$, Wang $Y$, Shao $C$, et al. COVID-19 infection: the perspectives on immune responses. Cell Death Differ 2020; 27: 1451-4.

5. Sadahiro $R$, Knight $B$, James $F$, et al. Major surgery induces acute changes in measured DNA methylation associated with immune response pathways. Sci Rep 2020; DOI: https://doi.org/ 10.1038/s41598-020-62262-x.

6. Tran K, Cimon K, Severn M, Pessoa-Silva CL, Conly J. Aerosol generating procedures and risk of transmission of acute respiratory infections to healthcare workers: a systematic review. PLoS One 2012; DOI: https://doi.org/10.1371/journal. pone.0035797.

7. Wang $W, X u Y$, Gao $R$, et al. Detection of SARS-CoV-2 in different types of clinical specimens. JAMA 2020; DOI: https:// doi.org/10.1001/jama.2020.3786.

8. Zou L, Ruan F, Huang M, et al. SARS-CoV-2 viral load in upper respiratory specimens of infected patients. N Engl J Med 2020; 382: 1177-9.

9. Yang $Y$, Yang $M$, Shen $C$, et al. Evaluating the accuracy of different respiratory specimens in the laboratory diagnosis and monitoring the viral shedding of 2019-nCoV infections. medRxiv 2020; DOI: https://doi.org/10.1101/2020.02.11.20021493.

10. Chan JF, Yip CC, To KK, et al. Improved molecular diagnosis of COVID-19 by the novel, highly sensitive and specific COVID19-RdRp/Hel real-time reverse transcription-PCR assay validated in vitro and with clinical specimens. J Clin Microbiol 2020; DOI: https://doi.org/10.1128/JCM.00310-20.

11. Cheng MP, Papenburg J, Desjardins $M$, et al. Diagnostic testing for severe acute respiratory syndrome-related coronavirus-2: a 
narrative review. Ann Intern Med 2020; DOI: https://doi.org/10. 7326/M20-1301.

12. Gudbjartsson DF, Helgason A, Jonsson H, et al. Spread of SARS-CoV-2 in the Icelandic population. N Engl J Med 2020; DOI: https://doi.org/10.1056/NEJMoa2006100.

13. Arons $M M$, Hatfield $K M$, Reddy SC, et al. Presymptomatic SARS-CoV-2 infections and transmission in a skilled nursing facility. N Engl J Med 2020; DOI: https://doi.org/10.1056/ NEJMoa2008457.

14. American Society of Anesthesiologists; Anesthesia Patient Safety Foundation. The ASA and APSF Joint Statement on
Perioperative Testing for the COVID-19 Virus. Available from URL: https://www.asahq.org/about-asa/newsroom/news-releases/ 2020/04/asa-and-apsf-joint-statement-on-perioperative-testingfor-the-covid-19-virus (accessed May 2020).

15. Government of Ontario. How Ontario is Responding to COVID19. Available from URL: https://www.ontario.ca/page/howontario-is-responding-covid-19 (accessed May 2020).

Publisher's Note Springer Nature remains neutral with regard to jurisdictional claims in published maps and institutional affiliations. 